\title{
MODEL REFERENCE ADAPTIVE CONTROL FOR TELEMANIPULATION
}

\author{
NÁNDOR FINK *1 \\ ${ }^{1}$ Department of Mechatronics, Optics and Engineering Informatics, Budapest University of Technology and \\ Economics, Bertalan Lajos utca 4-6, Budapest, 1111, HUNGARY

\begin{abstract}
A 1-DOF (degree-of-freedom) telemanipulation system is presented in this paper. The paper focuses on disturbance compensation of the haptic force feedback. The master and slave devices are connected via serial ports. The mechanism, which is applied as a human interface device, is subject to perceptible internal friction that must be eliminated. As a result, the operator would only feel the force feedback from the manipulated environment. The main contribution of this paper is the presentation of the telemanipulation device with a model reference adaptive control that compensates for the friction force using a direct model-based sliding mode algorithm.
\end{abstract}

Keywords: telemanipulation, friction compensation, sliding mode control

\section{INTRODUCTION}

In connection with the rapid spread of the Internet over the past few years, research about CSCW (ComputerSupported Cooperative Work) technology is being vehemently conducted. It is expected that this network-based technology will make collaboration in connection with human intellectual activities between distantly connected people easier. In the field of CSCW technology, the data to be processed are only images, sounds and other data for computers, little attention has been paid to multi-modal collaboration including physical contact. Our focus concerns networked multi-modal collaboration especially between those including haptics.

A collaboration tool that facilitates a better connection between laboratories, offices and factories in the manufacturing industry has become necessary [1-3].

Research into the bilateral control of master-slave manipulators was conducted in 1992. In [4] a 1-DOF telemanipulation system was tested. The research covered the dynamics of the human operator as well as the device. Three levels of ideal responses were determined, in all cases a force signal to the master arm was the input. The position of the two arms was identical in the first level, whereas the force response of the two arms was identical in the second, and both responses were identical in the third.

In [5] a four-channel control architecture was examined. Four models were tested on a teleoperation system, namely on an admittance-admittance, impedance-admittance, admittance-impedance and impedance-impedance pair. Based on the types of

\footnotetext{
*Correspondence: finknandor@mogi.bme.hu
}

model, a one-one suggestion was made for the control architecture in each case, namely Position-Position, Position-Force, Force-Position and Force-Force.

In addition to the basic criteria of stability, the ease of usability becomes increasingly important. To ensure comfortable and ergonomic telemanipulation, the operator should control the slave device more smoothly and precisely. Furthermore, the elimination of perturbations in all circumstances is necessary. For this purpose, the design of a model-reference adaptive control with a sliding mode friction compensator is promising.

\section{Theoretical background of the experi- ment}

\subsection{Sliding mode-based disturbance elimina- tion}

The main concept is the design of a reduced-order state observer for a partially perturbed linear system with infinite gain. The traditional roles of the system and observer are exchanged. The system is forced to follow the states of the unperturbed ideal model. Infinite gain is ensured by a sliding mode.

Consider the following partially perturbed linear system that consists of external disturbances and uncertain parameters which satisfy the so-called Drazenovic condition, written in the regular form of a state equation,

$$
\begin{aligned}
& \frac{\mathrm{d}}{\mathrm{d} t}\left[\begin{array}{c}
\mathbf{x}_{1} \\
\mathbf{x}_{2}
\end{array}\right]=\left[\begin{array}{cc}
\overline{\mathbf{A}}_{11} & \overline{\mathbf{A}}_{12} \\
\overline{\mathbf{A}}_{21}+\Delta \mathbf{A}_{21} & \overline{\mathbf{A}}_{22}+\Delta \mathbf{A}_{22}
\end{array}\right]\left[\begin{array}{l}
\mathbf{x}_{1} \\
\mathbf{x}_{2}
\end{array}\right] \\
& +\left[\begin{array}{c}
0 \\
\overline{\mathbf{B}}_{2}+\Delta \mathbf{B}_{2}
\end{array}\right] \mathbf{u}^{0}+\left[\begin{array}{c}
0 \\
\mathbf{E}_{2}
\end{array}\right] f(t)
\end{aligned}
$$


where $\mathbf{x}_{1} \in \mathbb{R}^{n-m}$ denotes the vector of non-perturbed state variables, $\mathbf{x}_{2} \in \mathbb{R}^{m}$ stands for the vector of perturbed state variables, $\mathbf{u}^{0} \in \mathbb{R}^{m}$ represents the input of the real system, $\overline{\mathbf{A}}_{i j}(i, j=1,2)$ and $\overline{\mathbf{B}}_{2}$ are the nominal or desired (ideal) matrices of the system, respectively, $\Delta \mathbf{A}_{2 j}(j=1,2)$ and $\Delta \mathbf{B}_{2}$ denote the bounded parameter perturbations, and $f(t)$ stands for the bounded external disturbance. The perturbed state variables are estimated by a discontinuous observer [6]

$$
\frac{\mathrm{d}}{\mathrm{d} t} \widehat{\mathbf{x}}_{2}=\overline{\mathbf{A}}_{21} \mathbf{x}_{1}+\overline{\mathbf{A}}_{22} \widehat{\mathbf{x}}_{2}+\overline{\mathbf{B}}_{2}\left(\mathbf{u}^{0}+\boldsymbol{\nu}\right)
$$

where $\nu$ denotes the discontinuous term. Let us design the following sliding surface:

$$
\boldsymbol{\sigma}=\left[\begin{array}{ll}
\mathbf{I} & -\mathbf{I}
\end{array}\right]\left[\begin{array}{l}
\mathbf{x}_{2} \\
\widehat{\mathbf{x}}_{2}
\end{array}\right]=0
$$

where $\mathbf{I} \in \mathbb{R}^{m \times m}$ represents the identity matrix and $\boldsymbol{\sigma} \in$ $\mathbb{R}^{m}$ stands for the distance from the surface. $\sigma$ must tend to zero. Let us calculate the elements of the discontinuous term $\nu$ in following way:

$$
\nu_{i}=G_{i} \operatorname{sign}\left(\sigma_{i}\right),
$$

where $G_{i}$ is the gain of the sliding mode controller. The implementation of a sliding mode means that the signs of $\sigma_{i}=0$ and $\nu_{i}$ change at an infinitely high frequency [7]. $\boldsymbol{\nu}$ can be substituted by its mean value denoted by $\boldsymbol{\nu}_{\text {eq }}$. By comparing the second line of Eq. 1 and Eq. 2:

$$
\begin{aligned}
\left(\overline{\mathbf{B}}_{2}+\Delta \mathbf{B}_{2}\right) \boldsymbol{\nu}= & \Delta \mathbf{A}_{21} \mathbf{x}_{1}+\left(\overline{\mathbf{A}}_{22}+\Delta \mathbf{A}_{22}\right) \mathbf{x}_{2} \\
& -\overline{\mathbf{A}}_{22} \widehat{\mathbf{x}}_{2}+\Delta \mathbf{B}_{2} \mathbf{u}^{0}+\mathbf{E}_{2} f(t)
\end{aligned}
$$

According to Eq. 5, $\nu$ can be used to estimate the perturbation. As a result, the response of the perturbed system in terms of $\mathbf{u}-\boldsymbol{\nu}$ will be identical to that of the unperturbed ideal system, $\mathbf{u}$.

The main problem concerning the sliding mode is the chattering caused by the infinitely alternating frequency of $\nu$. To avoid uncontrolled resonances of the unmodeled dynamics of the real system, $\boldsymbol{\nu}$ is substituted by $\boldsymbol{\nu}_{\text {eq }}$ (the continuous equivalent of $\nu$. In practice, it is impossible to calculate the equivalent control $\boldsymbol{\nu}_{\text {eq }}$ precisely, but it can be estimated by a low-pass filter for $\boldsymbol{\nu}$ as shown in Fig. 1, where two loops can be seen.

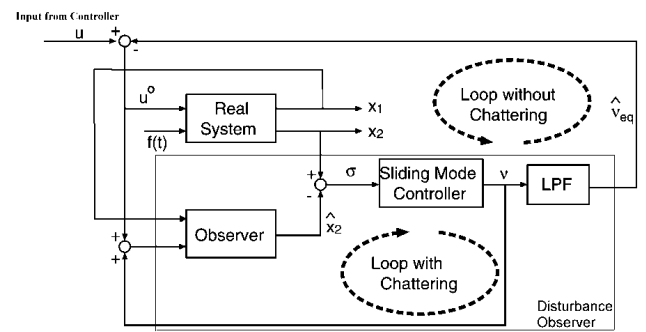

Figure 1: Sliding mode-based disturbance compensation.

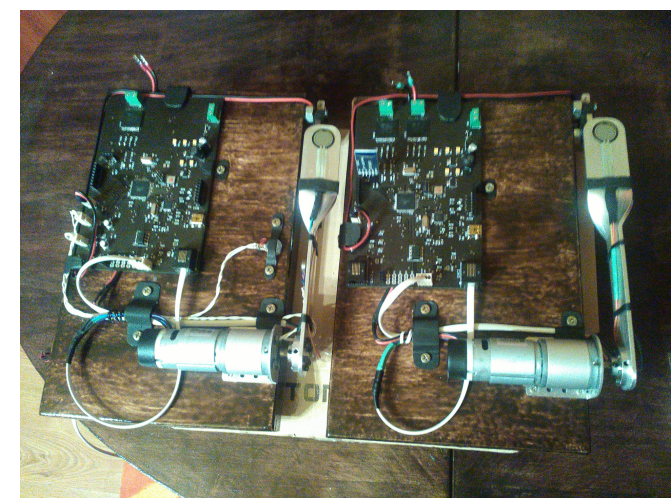

Figure 2: The haptic device.

The observer - sliding-mode control loop is calculated by the computer and should be as fast as possible to achieve an ideal sliding mode $[8,9]$. Since a reduced order observer is used, $\mathbf{x}_{2}$ of the real system is measured in terms of the disturbance compensation. Of course, all state variables might be measured in the outer control loop. $\widehat{\boldsymbol{\nu}}_{\text {eq }}$, the estimation of $\boldsymbol{\nu}_{\text {eq }}$, is added to the control signal of the outer control loop.

\section{Application}

\subsection{Tuning the disturbance compensation}

The master device consists of a DC motor and an arm (Fig. 2). The slave device is identical to the master device. Their roles are interchangeable. The arm is not rigid since the force is measured by strain gauges. In the case of the ideal telemanipulation model, the master device is not subject to friction nor mass (inertia). Of course it is impossible to construct an ideal master device. The goal of the sliding mode-based disturbance compensation is to force the master device to follow a model subject to significantly reduced degrees of friction and inertia. The model of the master and slave devices is shown in Fig. 3. The position of the motor is controlled by a simple Proportional Derivative (PD) controller. The results of three simulations were compared:

- PD controller with the parameters of the real motor

- PD controller with the parameters of the desired motor

- PD controller with the parameters of the real motor which were modified by the addition of slidingmode disturbance compensation.

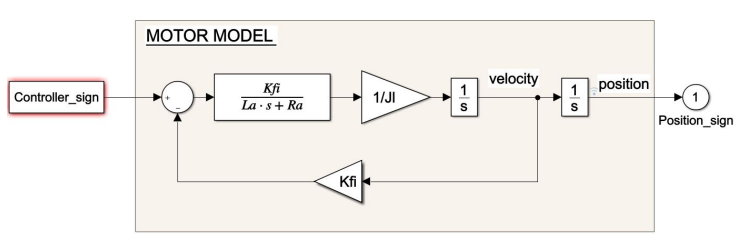

Figure 3: Model of the master and slave devices. 


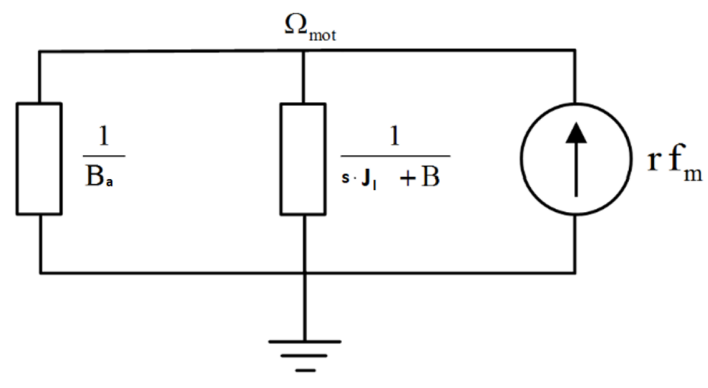

Figure 4: Model of the feedback force.

The main goal of this chapter is to tune the disturbance compensation component. If the results of the last two simulations are similar, sliding-mode disturbance compensation can be used in a bilateral telemanipulation system.

The parameters used in the model are calculable. A 1-DOF telemanipulation system was studied where the effect of the feedback force of the master arm was modelled [10] as follows (Fig. 4):

$$
\begin{gathered}
\frac{\boldsymbol{\Omega}_{\mathrm{mot}}}{\mathbf{r} \cdot \mathbf{f}_{m}}=\frac{1}{s \cdot J_{l}+B+B_{a}} \\
\frac{\Omega_{\mathrm{mot}}}{f_{m}}=\frac{\frac{r}{B+B_{a}}}{s \cdot \frac{J_{l}}{B+B_{a}}+1}=\frac{\frac{r}{B_{m}}}{s \cdot T+1},
\end{gathered}
$$

where $s$ is a complex variable and $T$ denotes the time constant of the manipulator:

$$
T=\frac{J_{1}}{B_{m}}
$$

$J_{l}$ is calculated according to:

$$
J_{\mathrm{l}}=J_{\mathrm{r}}+J_{\mathrm{tr} 2}+J_{\mathrm{a}},
$$

and represents the resultant moment of inertia, $J_{\mathrm{r}}$ stands for the inertia of the rotor, $J_{\mathrm{tr} 2}$ is the transmission inertia and $J_{\mathrm{a}}$ denotes the inertia of the arm.

$$
\mathbf{B}_{\mathrm{m}}=B+B_{\mathrm{a}}
$$

$\mathbf{B}_{\mathrm{m}}$ represents the resultant damping, $B$ the mechanical damping and $B_{\mathrm{a}}$ the damping caused by the armature resistance.

$$
B_{\mathrm{a}}=\frac{K_{\mathrm{fi}}^{2}}{R_{\mathrm{a}}}
$$

$K_{\mathrm{fi}}$ denotes the torque constant of the motor and $R_{\mathrm{a}}$ its resistance. $\Omega_{\text {mot }}$ stands for the angular speed of the motor, $f_{\mathrm{m}}$ the force- and $\mathbf{r}$ the vector of the lever arm with regard to the torque of the motor.

In this experiment, the following constants were used for the aforementioned parameters: $K_{\mathrm{fi}}=0.1222$ $[\mathrm{Nm} / \mathrm{A}], R_{\mathrm{a}}=80[\Omega], L_{\mathrm{a}}=0.0011[\mathrm{H}]$ and $J_{\mathrm{l}}=0.12$ $\left[\mathrm{kg} \mathrm{m}^{2}\right]$.

The model of the ideal motor took friction into account (Fig. 5), however, the other models accounted for this separately.

\subsection{Design of the disturbance compensation component}

In terms of human sensation, the electrical time constant of the system (the inductance of the DC motor) is negligible. Two state variables are used: the position of the arm $\varphi$ and the angular speed $\omega$. The system equation must be written in the same form as (Eq. 1) where:

$$
\frac{\mathrm{d}}{\mathrm{d} t}\left[\begin{array}{c}
\varphi \\
\omega
\end{array}\right]=\left[\begin{array}{cc}
0 & 1 \\
0 & a_{\text {motor }}
\end{array}\right]\left[\begin{array}{c}
\varphi \\
\omega
\end{array}\right]+\left[\begin{array}{c}
0 \\
b_{\text {motor }}
\end{array}\right] \mathbf{u}^{0},
$$

$a_{\text {motor }}$ and $b_{\text {motor }}$ are the two perturbed parameters. No external disturbance is applied. The observer is designed for the state variable $\omega$.

It should be noted that in the case of the ideal system, friction is accounted for in the model. The ideal parameters were selected in terms of friction and inertia. It is important to choose the ideal parameters wisely because these two parameters will determine the trajectory followed by the position signal of the compensated motor. If the values of the ideal parameters are too unrealistic, the compensated signal would not be able to follow the trajectory! In this simulation, the following parameters were used:

$$
J_{l_{-} \mathrm{i}}=\frac{J_{1}}{12} \quad B_{\mathrm{m}_{-} \mathrm{i}}=\frac{B_{\mathrm{m}}}{5}
$$

where $J_{l_{-} i}$ denotes the inertia with regard to the model of the ideal motor and $B_{\mathrm{m} \_\mathrm{i}}$ stands for the coefficient of friction concerning the model of the ideal motor.

The compensation component consists of the observer with the following transfer function:

$$
\frac{b_{\text {motor }}}{s+a_{\text {motor }}}
$$

where $a_{\text {motor }}$ is calculated by:

$$
a_{\text {motor }}=\frac{B_{\mathrm{m} \_\mathrm{i}}}{J_{l_{\_} \mathrm{i}}}-\frac{K_{\mathrm{fi}}^{2}}{R_{\mathrm{a}} \cdot J_{l_{-} \mathrm{i}}}
$$

and $b_{\text {motor }}$ by:

$$
b_{\text {motor }}=\frac{K_{\mathrm{fi}}}{R_{\mathrm{a}} \cdot J_{l_{\_} \mathrm{i}}}
$$

Then a PD position controller is added to each model of a motor (Fig. 5) and the component concerning the slidingmode compensator connected to the first real model of a motor (Fig. 6).

An estimated value of the velocity is specified in this section which will be compared with that of the actual velocity. This will be the input for the component of the sliding-mode controller that consists of a signum function and a gain block. From here a positive feedback was applied from the output of the sliding-mode controller to the input of the observer, and the output of the slidingmode controller filtered by a $3^{\text {rd }}$ order low-pass filter as the output of the whole compensation component (Fig. $6)$. 


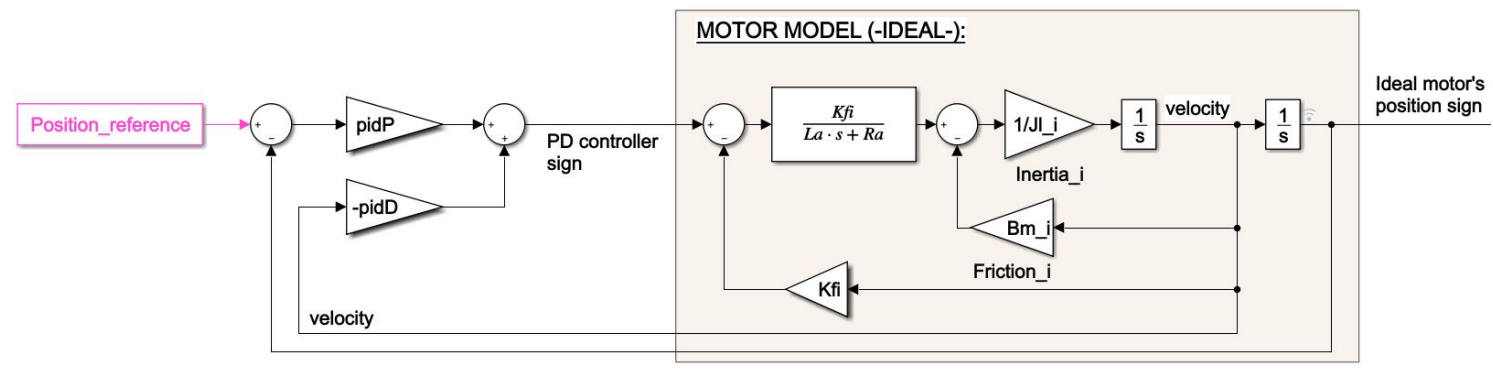

Figure 5: Model of the ideal motor with the application of a PD controller.

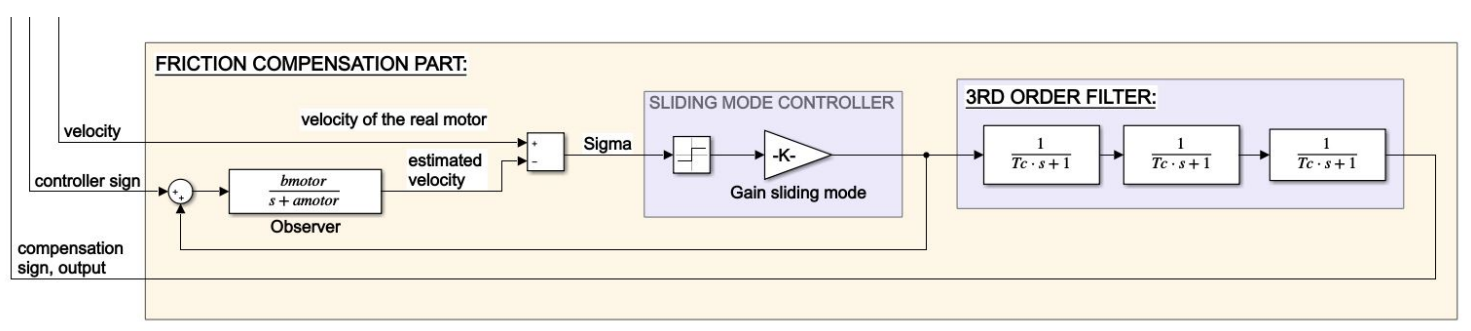

Figure 6: The component with regard to the compensation of friction.

\subsection{Calibration of the model}

To specify the parameters of the system, first the aforementioned model must be implemented. In the first case, a position controller was used for tuning (Fig. 7). It is worth noting that the position signal of the real motor reaches its final value by oscillation and overshooting, while the graphs of the ideal motor and the compensated motor are smoother. It has to be mentioned that the PD controller is not optimised for a stand-alone task, rather for the compensation. In this experiment the following constants were used for the aforementioned parameters: pidP $=190$, pidP $=60$ and Gain $_{\text {sliding }}=30,000$.

In the case of a 1-second-long simulation, the difference between the position trajectory of the ideal and compensated motors is visible (Fig. 8).

In Fig. 9 it is visible that the sliding-mode controller is inactive for about 0.2 seconds which is the time necessary for the controller to reach the sliding surface.

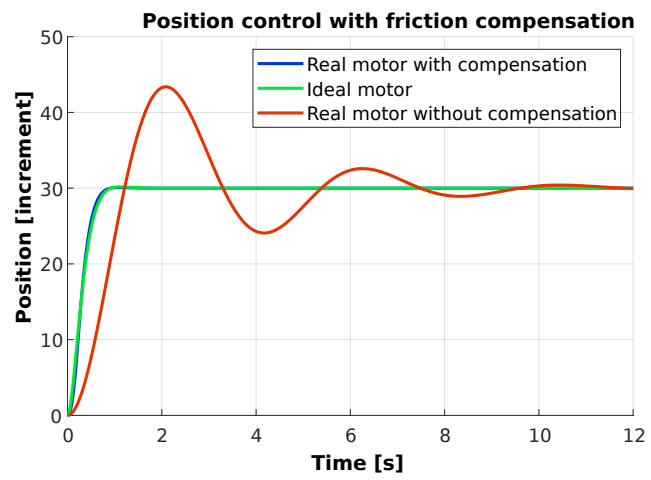

Figure 7: The compensated signal follows the stated trajectory.
Between -0.04 and +0.04 the active sliding-mode becomes visible (Fig. 10). There is a noticeable level of chattering originating from the output signal of the sliding-mode controller (Fig. 11). This was eliminated by the $3^{\text {rd }}$ order low-pass filter (Fig. 12).

\subsection{Verification of the model}

Subsequently, the calibration component follows on from verification of the model. The former simulations were run under nearly ideal conditions, but now a relevant Coulomb friction will be added to the system as well as an additional step function load. In Fig. 13 the supplemented system is shown only for the model of the motor that compensated for friction using real parameters (the models of the ideal and simple real motors were excluded to save space).

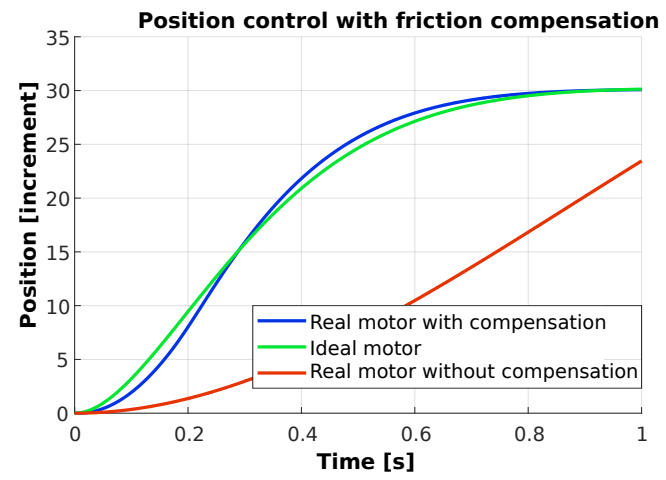

Figure 8: The difference between the position signals of the ideal and compensated motors. 


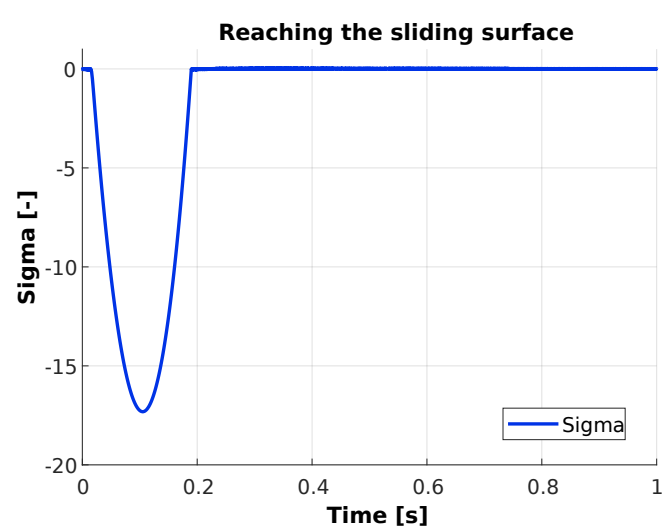

Figure 9: Reaching the sliding surface.

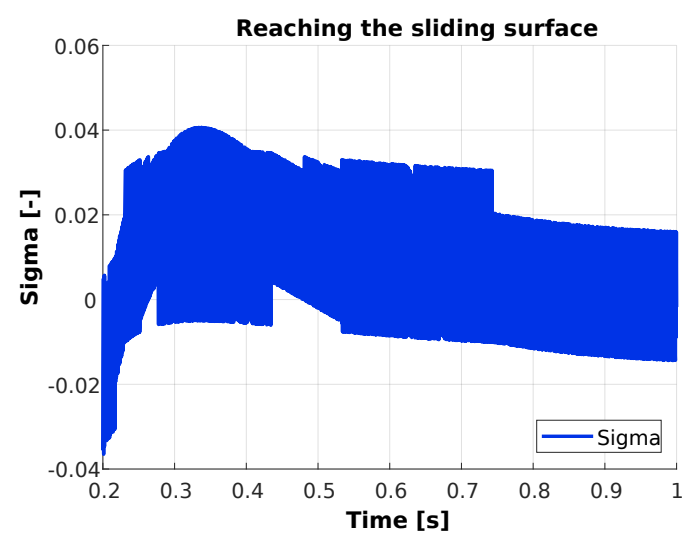

Figure 10: After reaching the sliding surface, the sliding mode becomes active.

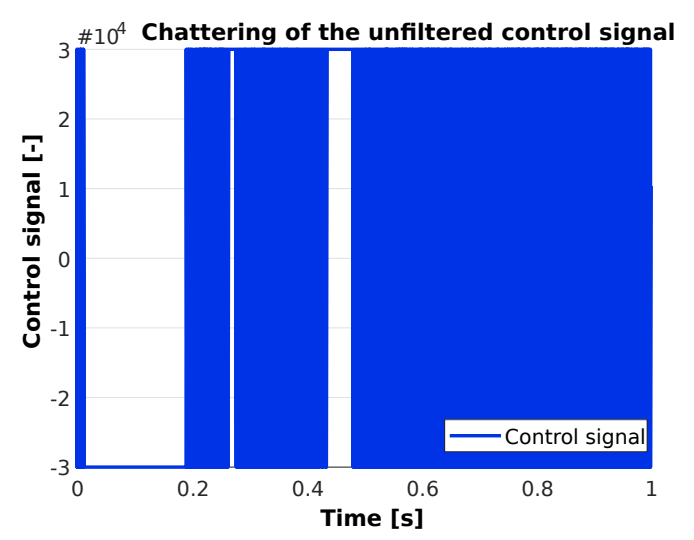

Figure 11: Chattering originating from the output of the sliding-mode controller.

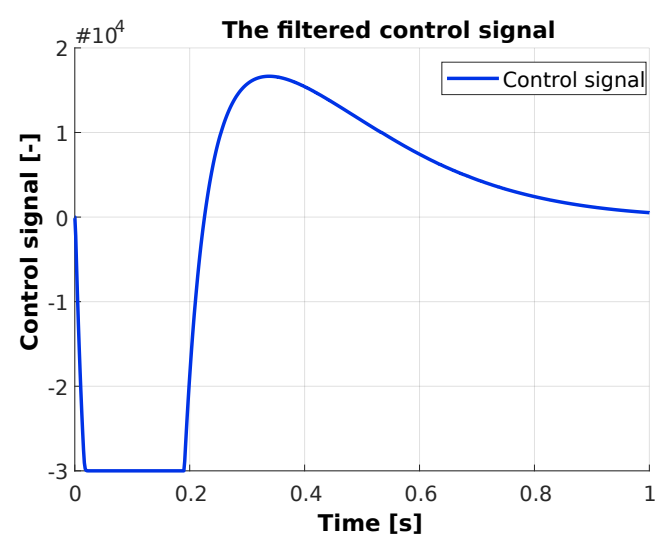

Figure 12: The filtered signal of the sliding-mode controller.

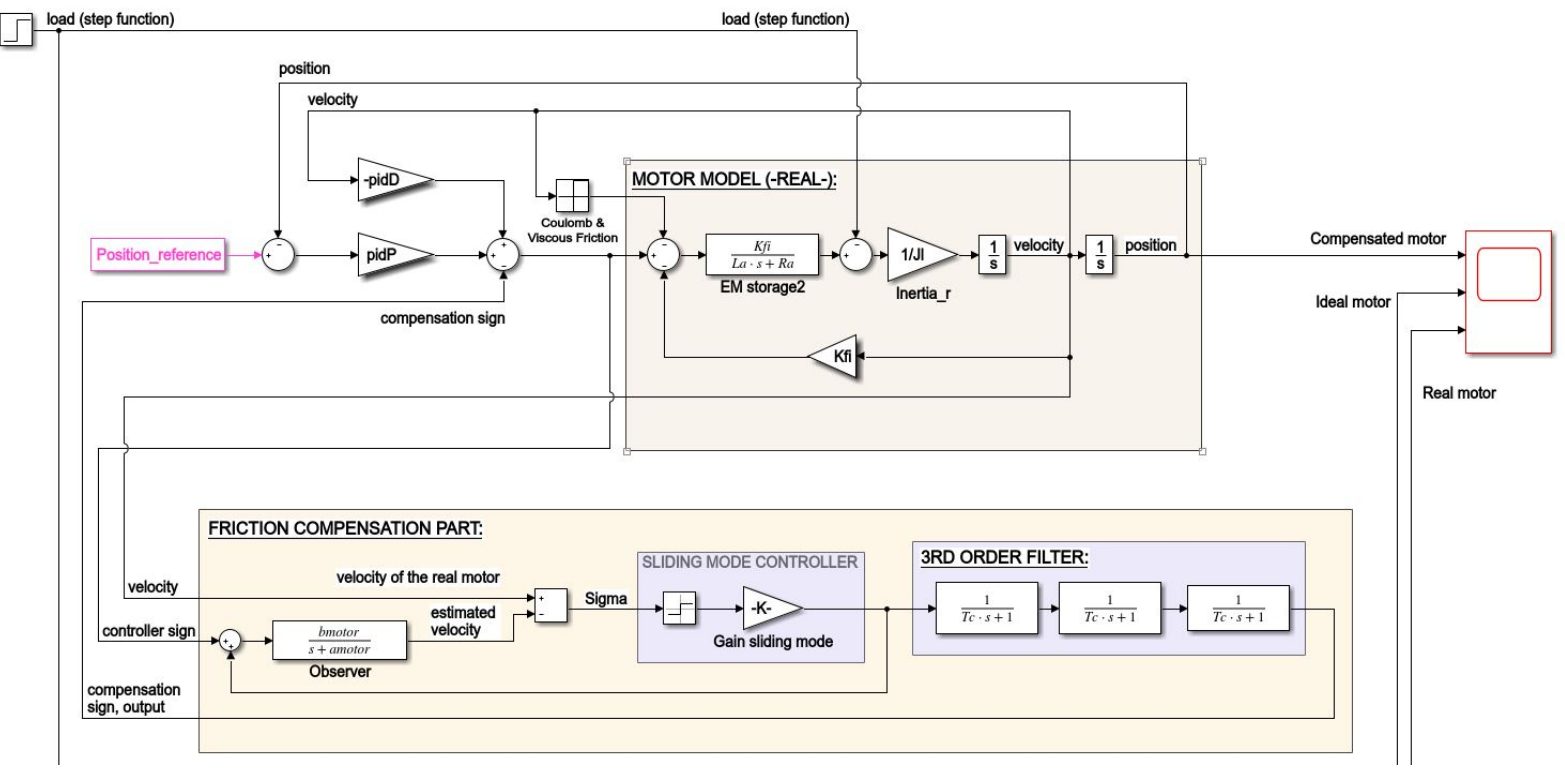

Figure 13: Full model of the compensated motor subjected to calibration. 


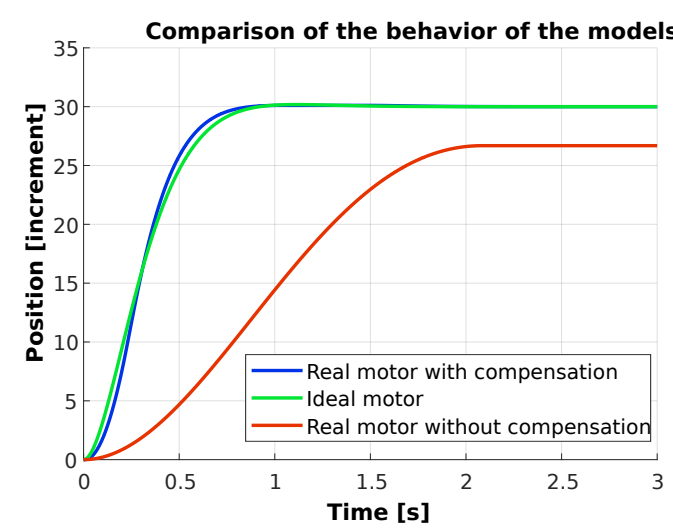

Figure 14: Position signals after Coulomb friction was accounted for in the system.

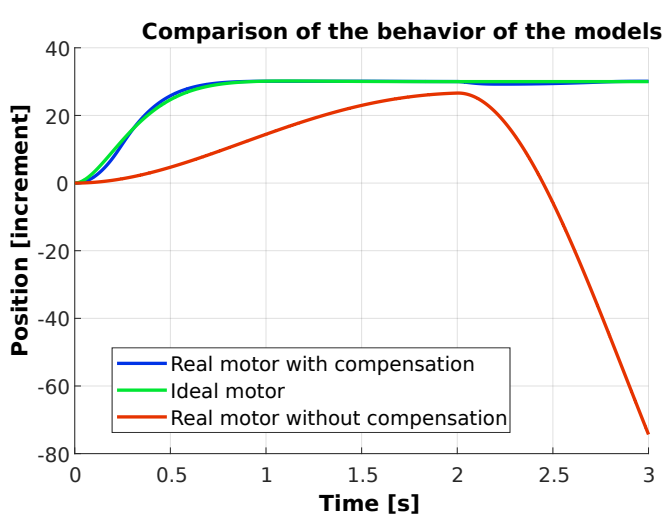

Figure 15: An additional load added to the system.

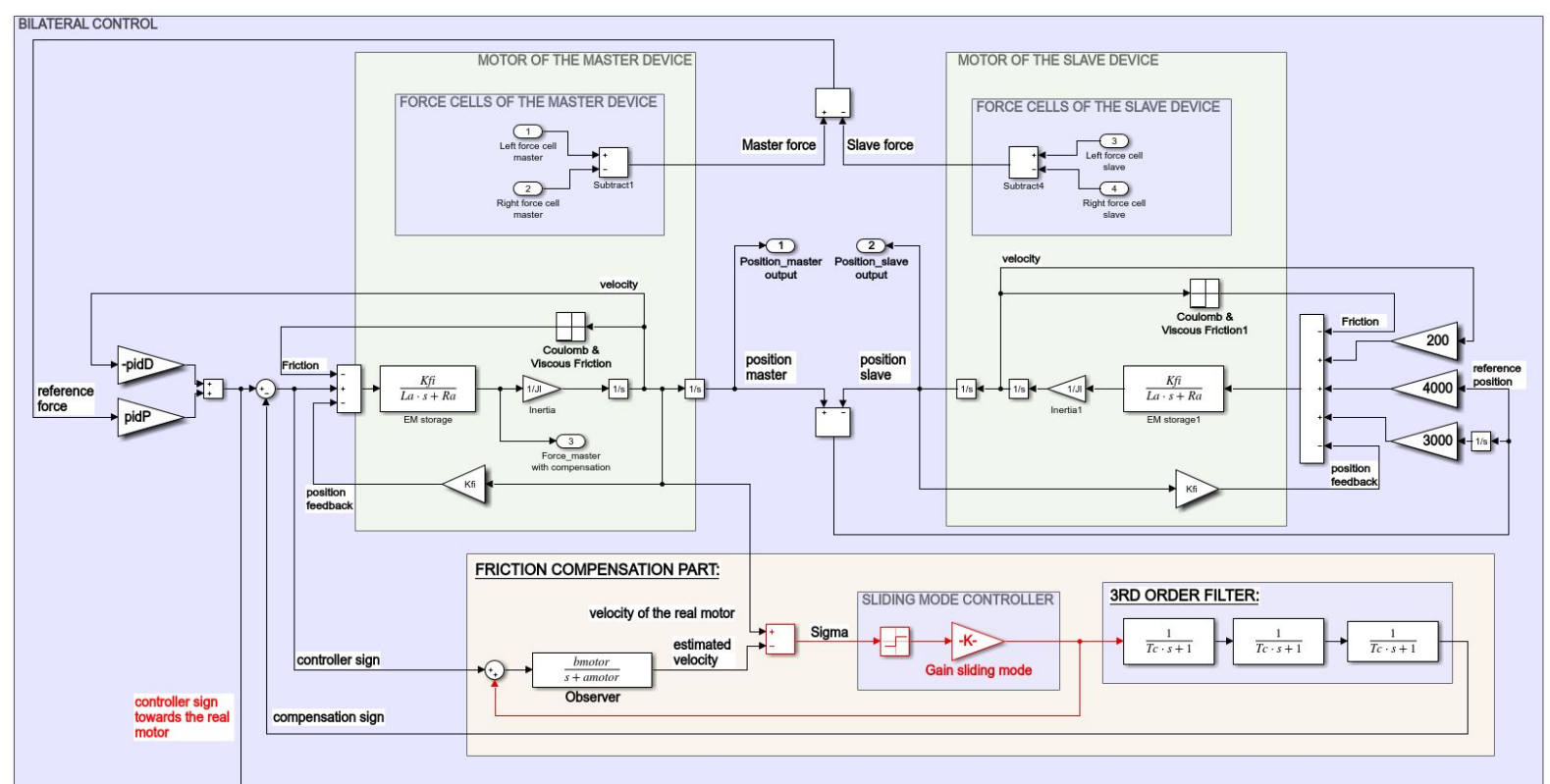

Figure 16: Bilateral control model.

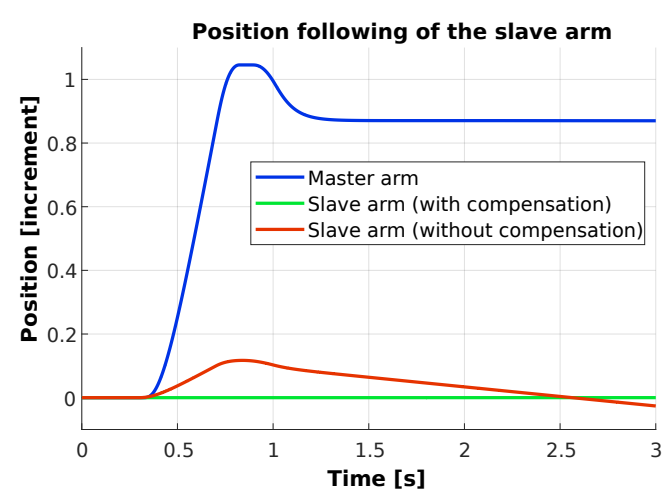

Figure 17: The same parameters applied to the slave arm failed to yield results.

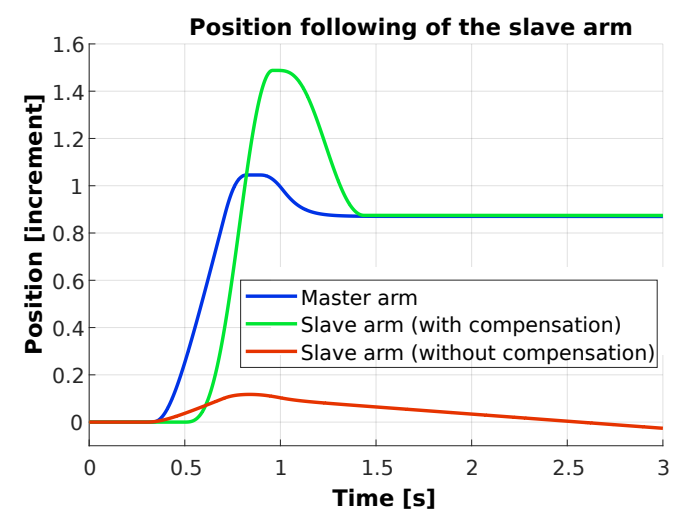

Figure 18: Position signal following of the bilateral system. 


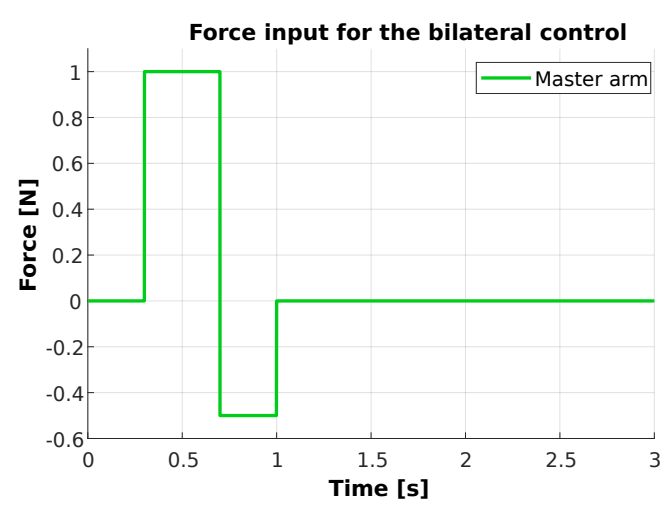

Figure 19: Force input to the bilateral control.

In Fig. 14 the position signals are shown after the addition of the Coulomb friction to the system. This new component has a stabilizing effect on the oscillation of the real motor which is greater than can be compensated for without the introduction of a permanent error. However, the behaviour of the motor compensated for by friction remains unchanged. The values of the Coulomb friction and viscous friction used in this experiment were 2,200 and 0.3 , respectively. Then after 2 seconds an additional load was added to the system (Fig. 15). It was noticeable that when the position signal of the normal motor started to rapidly decrease, the model compensated for by friction adapted to the new circumstances. The value of the load added after $2 \mathrm{~s}$ was 42 .

\section{Results and Discussion}

\subsection{Final model}

Finally, bilateral haptic control was realized (Fig. 16). The model of the two force cells on the real-life device was added to the system by applying the same logic as used on the real. Each model of the motor was extended by a left- and right-force block.

In terms of bilateral control, the difference between the force blocks of each joystick was compared, which was also the input of the force controller of the master arm. Subsequently, the position of the master arm was compared to that of the slave arm, which was the input of the position controller of the slave arm (Fig. 16). It would be illogical to apply the same parameters to the position controller as to the one that accounts for compensation because without the component of compensation the friction force would prevent the proper position from being reached (Fig. 17).

Nevertheless, if an individually tuned proportionalintegral-derivative (PID) controller is connected to the component responsible for position control of the system, the signal that results would be visible (Fig. 18). In this case, the input signal was applied to the right force cell of the master arm in a shape as is shown in Fig. 19.

It is impossible to eliminate the entire effects of friction and inertia on the mechanical construction, but they can be reduced significantly. The magnitude of the friction force exceeded the limit suitable for a smooth, comfortable operation. Compensation aims to make the system behave like an ideal model subject to a minimal level of friction. Due to the compensation, the operator can move the master joystick with ease.

Over a series of experiments, classical and sliding mode-based model reference adaptive control methods were compared. The applications of these methods with regard to compensation for friction are published in $[11,12]$.

It is crucial to identify an optimal adaptation parameter which facilitates rapid adaptation but avoids overcompensation. In the event of overcompensation, the joystick moves randomly even in the absence of reference torque because of the measurement noise. As the size of the adaptation parameter increases, adaptation occurs more rapidly, however, the likelihood of random movements rises.

\section{Conclusion}

An experimental telemanipulation system was presented in this paper. The master device was a serial linked levertype haptic interface with force feedback. Even though it is impossible to eliminate the entire effects of friction and inertia on the mechanical construction, a reference model that accounts for minor levels of friction was designed. The system was forced to follow the reference model. In other words, the original dynamics of the master device were replaced by a virtual version which ensures a comfortable degree of manipulation for the operator.

\section{REFERENCES}

[1] Arai, F.; Sugiyama, T.; Fukuda, T.; Iwata, H.; Itoigawa K.: Micro tri-axial force sensor for 3D bio-micromanipulation, 1999 IEEE International Conference on Robotics and Automation, 1999 4, pp. 2744-2749 ISBN: 0-7803-5180-0 DOI: 10.1109/ROBOT.1999.774012

[2] Kwon, D-S.; Woo, K. Y.; Cho, H. S.: Haptic control of the master hand controller for a microsurgical telerobot system, 1999 IEEE International Conference on Robotics and Automation, 1999 3, pp. 1722-1727 ISBN: 0-7803-5180-0 DOI: 10.1109/ROBOT.1999.770357

[3] N. Ando, M. Ohta, H. Hashimoto: "Micro Teleoperation with Haptic Interface", Proceedings of the 2000 IEEE Int. Conf. on Industrial Electronics, Control and Instrumentation, pp. 13-18, 2000, 10.1109/IECON.2000.973119

[4] Yokokohji, Y.; Yoshikawa, T.: Bilateral control of master-slave manipulators for ideal kinesthetic coupling-formulation and experiment, IEEE T. Robotics Autom., 1994 10(5), 849-858 DOI: $10.1109 / 70.326566$ 
[5] Hashtrudi-Zaad, K.; Salcudean, S. E.: Analysis of control architectures for teleoperation systems with impedance/admittance master and slave manipulators, Int. J. Robotics Res., 2001 20(6), 419-445 DOI: 10.1177/02783640122067471

[6] Korondi, P.; Young, K. D.; Hashimoto, H.: Sliding mode based disturbance observer for motion control In: Proceedings of the 37th IEEE Conference on Decision and Control, (IEEE Press, New York, USA) 1998, pp. 1926-1927 ISBN: 0-7803-4394-8 DOI: 10.1109/CDC.1998.758595

[7] V. I. Utkin: Sliding Modes in Control and Optimization, Springer -Verlag, 1992, ISBN: 978-3-642-84379-2 DOI: 10.1007/978-3-642-84379-2

[8] Ando, N.; Korondi, P.; Hashimoto, H.: Development of micromanipulator and haptic interface for networked micromanipulation, IEEE/ASME T. Mechat., 2001 6(4), 417-427 DOI: 10.1109/3516.974855
[9] Korondi, P.; Young, K-K. D.; Hashimoto, H.: Discrete-time sliding mode based feedback compensation for motion control, 1996 IEEE International Workshop on Variable Structure Systems, 1996, pp. 237-242 ISBN: 0-7803-3718-2 DOI: 10.1109/VSS.1996.578577

[10] Lukács P.: 1 degree of freedom haptic device for telemanipulation, Master's Thesis, 2015

[11] Ando, N.; Ohta, M.; Hashimoto, H.: Micro teleoperation with parallel manipulator, 2000 IEEE/RSJ International Conference on Intelligent Robots and Systems, 2000 1, pp. 677-682 ISBN: 0-7803-6348-5 DOI: $10.1109 /$ IROS.2000.894682

[12] Korondi, P.; Szemes, P. T.; Hashimoto, H.: Sliding mode friction compensation for a 20 DOF sensor glove, J. Dyn. Sys., Meas., Control, 2000 122(4) 611-615 DOI: $10.1115 / 1.1317232$ 